\title{
Spatial horizons in amplitude and frequency modulation atomic force microscopy
}

\author{
Josep Font ${ }^{1, \ddagger}$, Sergio Santos ${ }^{2, \ddagger}$ Victor Barcons ${ }^{1}$, Albert Verdaguer ${ }^{3}$, Matteo Chiesa ${ }^{2,4, *}$
}

${ }^{1}$ Departament de Disseny i Programació de Sistemes Electrònics, UPC - Universitat Politècnica de Catalunya Av. Bases, 61, 08242 Manresa, Spain ${ }^{2}$ Laboratory of Energy and Nanosciences, Masdar Institute of Science and Technology, Abu Dhabi, UAE ${ }^{3}$ Centre d' Investigació en Nanociència i Nanotecnologia (CIN2) (CSIC-ICN), Esfera UAB, Campus de la UAB, Edifici CM-7, 08193-Bellaterra, Catalunya, Spain ${ }^{4}$ Dep. of Mechanical Engineering, Massachusetts Institute of Technology, 77 Massachusetts Avenue Cambridge, MA 02139-4307

*mchiesa@mit.edu

$\ddagger$ These authors contributed equally. 


\begin{abstract}
.
In dynamic atomic force microscopy (AFM) the cantilever is vibrated and its dynamics are monitored to probe the sample with nanoscale and atomic resolution. Amplitude and frequency modulation (AM and FM) atomic force microscopy have established themselves as the most powerful, robust and reliable techniques in the field. Nevertheless, it is still debatable whether one or the other technique is preferred in a given medium or experiment. Here, we quantitatively establish the limitations in resolution of one and the other technique by introducing the concept of space horizon $\mathrm{SH}$ and quantifying it. The SH is the limiting space boundary beyond which collective atomic interactions do not affect the detection parameters of a given feedback system. We show that while an FM feedback can resolve an atom where an AM feedback might fail, relative contrast is in fact equivalent for both feedback systems. That is, if the AM feedback could detect sufficiently small amplitude shifts and there was no noise, single atom imaging would be equivalent in $\mathrm{AM}$ and FM.
\end{abstract}

Keywords: AFM, frequency modulation, amplitude modulation, resolution, sensitivity 


\section{Introduction}

Atomic force microscopy (AFM) and optical and electron microscopy (OM) and (EM) have many characteristics and applications in common. It is however an intriguing and unique characteristic of the AFM that resolution and contrast so strongly depend on a physical parameter that might greatly vary from experiment to experiment. This is the curvature and state of the tip. In order to obtain atomic resolution, it is generally believed that the interaction between a single atom on the tip and another single atom on the sample is required to account for most of the total tip-sample force ${ }^{1}$. Thus, when high resolution is achieved with the use of relatively blunted tips, single asperity contacts are typically thought to be responsible ${ }^{2}$. The nature of AFM has also led to the coining of terms such as true atomic resolution, true molecular resolution and lattice resolution according to whether the atomic or molecular periodicity correlates with inter-atomic or molecular distances and the presence of atomic like defects with the characteristic intermolecular separations can be resolved or not ${ }^{3-5}$. The most powerful AFM modes, in terms of resolution, are the dynamic modes, and in particular, amplitude and frequency modulation AFM (AM and FM AFM) ${ }^{2-3,6}$; even though static modes might achieve atomic resolution they have severe limitations in many cases ${ }^{2}$. Nevertheless, dynamic modes add further complications to the interpretation of data due to the complex dynamic behavior of the cantilever when the tip interacts with the nonmonotonic, and sometimes non-continuous, tip-sample force during one oscillation cycle. Furthermore, there are several modes of that can be selected to operate the instrument. For example, in the non-contact mode, mechanical contact with the surface never occurs during one cycle ${ }^{6}$. In the attractive regime, intermittent contact might occur but the net force per cycle is attractive. In the repulsive regime, intermittent 
contact with the surface occurs and the net force per cycle is repulsive; for convenience only the non-contact and the repulsive regimes are discussed here. The non-contact and the repulsive regimes are typically controlled by the oscillation and free amplitude where small and large amplitudes lead to one and the other respectively. Here, we define oscillation amplitude as the perturbed amplitude and free amplitude as the unperturbed amplitude. Both the attractive and the repulsive regime have been shown to lead to atomic resolution ${ }^{2,4}$. Nevertheless, because of being less destructive, non-contact (nc) modes are gaining importance in the community over repulsive imaging ${ }^{3,7}$.

AM and FM are basically differentiated in terms of the feedback mechanism which is used to detect variations in the cantilever-dynamics due to differences in the tip-sample interaction; amplitude and frequency respectively ${ }^{6}$. The nature of the interaction itself is equivalent when the instruments are operated in the same environment and with the same cantilever-sample systems. The difference in sensitivity, in amplitude and frequency respectively, relative to differences in tip-sample forces, and the respective particular way in which the sample is tracked in one and the other modes, has nevertheless been shown to lead to strikingly different outcomes in terms of resolution and capabilities ${ }^{8-9}$. One and the other, each feedback mechanism is more suitable in (moderate or highly) damped (AM) or low damped (FM) environments ${ }^{6}$. FM is typically preferred for atomically resolving periodic lattices and AM is believed to cope better with challenging topography and large scan areas ${ }^{10}$. Moreover, while technical developments in one and the other modes keep making progress in both directions ${ }^{5-6,11-}$ ${ }^{13}$, the debate on the relative sensitivity of one and the other feedback modes is ongoing. Some have stated that experimental comparison seems to reflect the skill of the user rather than the limitations of a feedback mode and quantitative studies are thus 
lacking $^{10}$. Here, we calculate the spatial sensitivity limits of the AFM in terms of both frequency and amplitude and term this limits in sensitivity spatial horizons ( $\mathrm{SH}$ ). In short, the $\mathrm{SH}$ is the limiting area of interaction for which the dynamics of the cantilever lose sensitivity with respect to the atoms lying beyond it in terms of frequency and amplitude respectively (Fig. 1). We show that this concept can be used to discuss both the advantages and disadvantages of dynamic modes and interpret the limitations in spatial resolution and the problems arising with heterogeneous topography. In particular, we establish that the main characteristic that makes FM superior to AM, in terms of the detection of single atoms or single atomic defects, is the capacity of present technology to detect frequency variations.

\section{Results and discussion}

A schematic of a tip vibrating with amplitude $\mathrm{A}$ in the non-contact mode is shown in Fig. 1a. The minimum distance of approach is termed $d_{\min }$ where $d$ is the instantaneous tip-sample distance; mechanical contact occurs at $\mathrm{d}=\mathrm{a} 0$ where $\mathrm{a}_{0}$ is an intermolecular distance which implies that matter interpenetration cannot occur. We use $\mathrm{a}_{0}=0.165 \mathrm{~nm}^{14}$. The spatial horizon $\mathrm{SH}$ for this system is delimited by dashed lines. The $\mathrm{SH}$ is defined as the effective area for which the dynamics, either amplitude or frequency, are not affected by interactions due to the atoms lying beyond it. It is important to note that we can define whether the dynamics are affected by tip-sample interactions in terms of the sensitivity of a particular feedback system. That is, while, strictly speaking, the tip interacts with the infinite surface, only the interaction with atoms lying sufficiently close can affect the dynamics to the extent that feedbacks can detect them. This is what 
we terms sensitivity. The scheme in Fig. 1a shows that a radial distance $\mathrm{r}$ can be defined as the radius of the $\mathrm{SH}$ for each feedback system. One can then write $\mathrm{r}_{\mathrm{FM}}$ and $\mathrm{r}_{\mathrm{AM}}$ for the radial distance of each feedback. In Fig. 1b a similar scheme is shown depicting what happens to the SH when mechanical contact occurs; the contributions to SH might be include contact and non-contact interactions. We now proceed to quantify the sensitivity of the AM and FM feedbacks by describing the respective differences in $\mathrm{SH}$, i.e. $\mathrm{r}_{\mathrm{FM}}$ and $\mathrm{r}_{\mathrm{AM}}$.

(a)
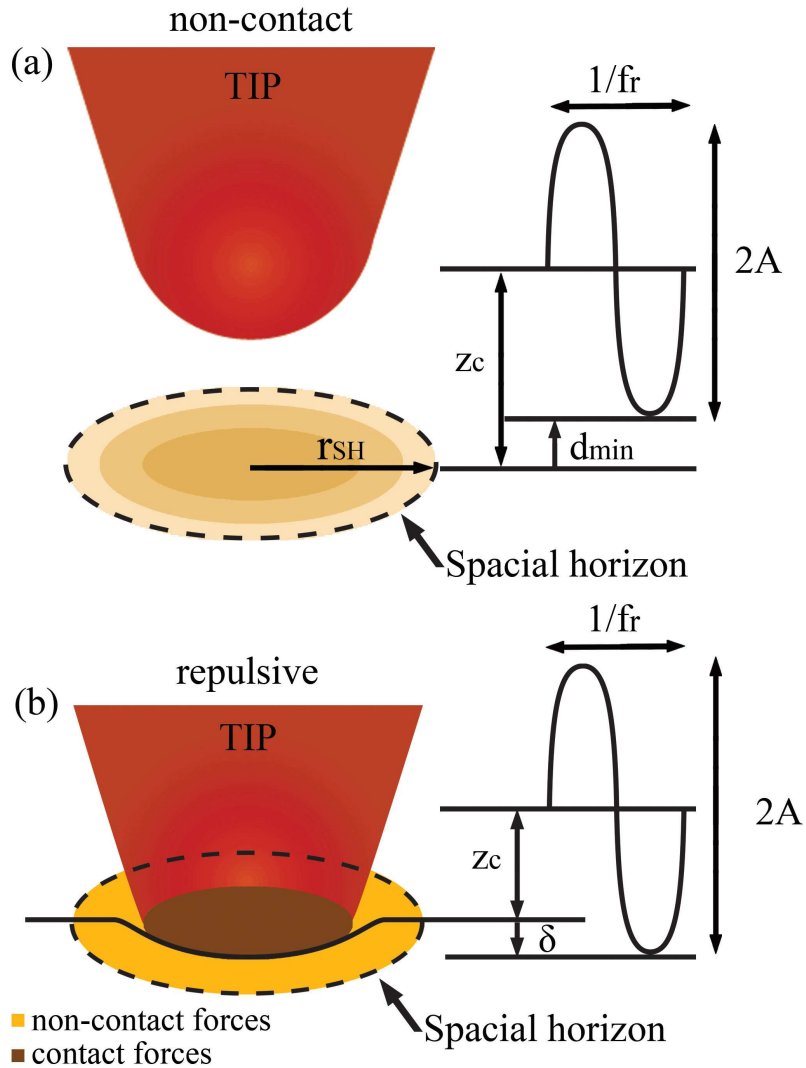

Figure 1. (a) Scheme of a tip vibrating in the non-contact mode where $d_{\min }>a_{0}$ and $\delta=0$. The Spatial Horizon ( $\mathrm{SH}$ ) is thus affected by long range forces only. The interactions occurring between the tip and the sample's atoms lying beyond the boundary established by the SH do not sufficiently affect the dynamics of the cantilever for the feedback to detect them. (b) Scheme of a tip vibrating in the repulsive regime where intermittent mechanical contact occurs $\mathrm{d}_{\min }>\mathrm{a}_{0}$ and $\delta>0$. The $\mathrm{SH}$ in this case is affected by both short range and long range forces. 
The interaction is prescribed (Fig. 1) by the instantaneous tip-sample distance $d$ and the equilibrium cantilever-sample separation $\mathrm{z}_{\mathrm{c}}$. The instantaneous tip position $\mathrm{z}$, as measured from $z_{c}$, is related to $d$ by the geometrical relationship $d=z+z_{c}$ and to $A$ by $\mathrm{z}=\mathrm{z}_{0}\left(\mathrm{z}_{\mathrm{c}}\right)+\mathrm{A}\left(\mathrm{z}_{\mathrm{c}}\right) \cos \left(\omega \mathrm{t}+\Phi\left(\mathrm{z}_{\mathrm{c}}\right)\right)$. The latter implies that only the first harmonic is taken into account. This is a good approximation when the Quality factor is high as in the case of the present study ${ }^{15}$. Here $\omega$ is the angular oscillation frequency and $\Phi$ is the phase lag relative to the drive force. Now, we write the equation of motion (1) with the understanding $^{6,16}$ that each feedback mode will alter parameters according to the prescribed frequency shift in FM and amplitude shift in AM that a use might $\operatorname{set}^{6}$. We write $^{6,17}$

$m \frac{d^{2} z}{d t^{2}}+\frac{m \omega_{0}}{Q} \frac{d z}{d t}+k z=F_{t s}+F_{0} \cos \omega t$

where the details of the parameters and its limits of application in ambient conditions are described elsewhere ${ }^{15,17}$. We consider for the net tip-sample force $F_{t s}$ a standard conservative potential which is well established and robustly tested in dynamic $\mathrm{AFM}^{6}$, ${ }^{10}$. This model consists of the long range van der Waals (vdW) forces $F_{a}$ and short range forces $\mathrm{F}_{\mathrm{DMT}}$ modeled with the Derjaguin-Muller-Toporov (DMT) model of contact mechanics $^{17-18}$

$$
\begin{array}{ll}
F_{a}(d)=-\frac{H R}{6 d^{2}} & \mathrm{~d}>\mathrm{a}_{0} \\
F_{D M T}(d)=-\frac{H R}{6 a_{0}^{2}}+\frac{4}{3} E^{*} \sqrt{R \delta^{3}} & \mathrm{~d} \leq \mathrm{a}_{0}
\end{array}
$$

where $\mathrm{H}$ is the Hamaker constant, $\mathrm{E}^{*}$ is the effective elastic modulus of the tip and the sample and $\delta$ is the instantaneous indentation (see Fig. 1b). 
We are now in the position to quantify SHs in FM and AM and their corresponding radii $r_{F M}$ and $r_{A M}$. Let us probe the pure nc mode and the repulsive mode of operation by prescribing a given set of operational and cantilever-sample parameters; $\mathrm{A}_{0}$ (free or unperturbed amplitude), $\mathrm{z}_{\mathrm{c}}$ (equilibrium cantilever-sample separation), $\mathrm{f}_{0}$ (natural frequency of oscillation), $\mathrm{Q}$ ( $\mathrm{Q}$ factor), $\mathrm{E}_{\mathrm{s}}$ (elastic modulus of the sample), $\mathrm{E}_{\mathrm{t}}$ (elastic modulus of the tip), $\mathrm{H}$ (Hamaker constant) and $\mathrm{R}$ (tip radius). In the nc mode, and for $\mathrm{AM}$, we set $\mathrm{A}_{0}=2 \mathrm{~nm}, \mathrm{z}_{\mathrm{c}}=2 \mathrm{~nm}, \mathrm{f}_{0}=300 \mathrm{kHz}, \mathrm{Q}=500, \mathrm{E}_{\mathrm{s}}=1.4 \mathrm{GPa}, \mathrm{E}_{\mathrm{t}}=120 \mathrm{GPa}, \mathrm{R}=5$ and $20 \mathrm{~nm}$. These parameters are typical of ambient imaging where the $\mathrm{Q}$ factor is such that both AM and FM feedback controllers can relatively easily operate ${ }^{6}$. Now, we set the drive frequency $\mathrm{f}$ in $\mathrm{AM}$ at the natural frequency of oscillation, i.e. $\mathrm{f}=\mathrm{f}_{0}$, and record the oscillation amplitude A that follows from the dynamics. This is done by numerically solving the equation of motion (1). Note that this is equivalent to standard experimental practice in the AM mode of operation and corresponds to setting an initial free amplitude $\mathrm{A}_{0}$ and the required amplitude shift $\mathrm{A}-\mathrm{A}_{0}$ for imaging. We obtain $\mathrm{A}=1.73$ for $\mathrm{R}=5 \mathrm{~nm}$ (filled squares) and $1.52 \mathrm{~nm}$ for $\mathrm{R}=20 \mathrm{~nm}$ (filled triangles) when all the atoms in the infinite surface are accounted for. These values are taken as references and termed $A_{\text {ref }}$ where ref stands for reference or infinite surface. Now, in order to find $r_{A M}$ atoms are removed from the surface in a radial fashion and the new oscillation amplitude $A(r)$ is recorded as a function of radial distance $r$ (see Fig. 1); the difference $\mathrm{A}(\mathrm{r})-\mathrm{A}_{\text {ref }}$ results as a consequence of atoms removal. The results of this procedure are shown in Fig. 2a where in the vertical axis $\mathrm{A}(\mathrm{r})-\mathrm{A}_{\text {ref }}$ has been plotted. In the horizontal axis the radial distance $\mathrm{r}$ is plotted in $\mathrm{nm}$ and in a logarithmic scale. The values $\mathrm{A}_{\text {ref }}$, for $\mathrm{R}=5$ and $20 \mathrm{~nm}$ respectively, can then be set in the FM system; the same parameter values are kept for a fair comparison. Note that setting an initial value of $\mathrm{A}$ for scanning, i.e. in this case $A=A_{\text {ref }}$, is standard procedure in FM. That is, in FM a value of 
amplitude $A=A_{0}$ and a required frequency shift $f-f_{0}$ are set. As the tip interacts with the sample the required shift in frequency is obtained by varying $\mathrm{z}_{\mathrm{c}}$. This procedure is carried out by the feedback system. However, in the FM simulations we set the same values of $\mathrm{z}_{\mathrm{c}}$ and $\mathrm{A}$ as those previously obtained in $\mathrm{AM}$ in order to compare the sensitivity for a given set of parameters. From the dynamics, the resonant frequency then shifts to f; this is required in FM for feedback control. For the infinite surface $\mathrm{f}=299835.85$ for $\mathrm{R}=5 \mathrm{~nm}$ (outlined squares) and $299748.32 \mathrm{~Hz}$ for $\mathrm{R}=20 \mathrm{~nm}$ (outlined triangles). These values are taken as frequency references $f_{\text {ref }}$ as before. Then, one can follow the same procedure as in AM and start removing atoms in the radial direction of the sample in order to obtain $\mathrm{f}(\mathrm{r})$ or $\mathrm{f}(\mathrm{r})-\mathrm{f}_{\text {ref. }}$. In Fig. $2 \mathrm{a}$ the results obtained in the noncontact mode are shown for the AM (filled markers) and FM (outlined markers) respectively. The calculations can also be carried out in the repulsive regime (Fig. 2b) by using a different set of operational parameters, i.e. $\mathrm{A}_{0}=50 \mathrm{~nm}$ and $\mathrm{z}_{\mathrm{c}}=30 \mathrm{~nm}$ (other parameters as above). The results are $\mathrm{A}_{\text {ref }}=32.41$ and $31.87 \mathrm{~nm}$ for $\mathrm{R}=5$ (filled squares) and $20 \mathrm{~nm}$ (filled triangles) and $\mathrm{f}_{\text {ref }}=300317.08$ and $300308.63 \mathrm{~Hz}$ for $\mathrm{R}=5$ (outlined squares) and $20 \mathrm{~nm}$ (outlined triangles) respectively. Finally, in order to define the $\mathrm{SH}$ from these figures, the minimum values of detectable amplitude $\mathrm{A}$ and frequency $\mathrm{f}$ shifts in $\mathrm{AM}$ and FM respectively need to be taken into account. We term these $\delta(\mathrm{A})$ and $\delta(\mathrm{f})$ respectively and take $\delta(\mathrm{A})=0.05 \mathrm{~nm}$ and $\delta(\mathrm{f})=0.1 \mathrm{~Hz} ; \quad \delta(\mathrm{A})=0.05$ is a reasonable experimental value for amplitude detection at $300 \mathrm{~K}$ and $\delta(\mathrm{f})=0.1 \mathrm{~Hz}$ is close to the frequency detection limits when imaging ${ }^{6}$. Note that these values are being used for easiness in the comparisons. That is, the actual values might depend on the setup and state of the art of the technology but the approach can be used for any given pair of $\delta(\mathrm{A})$ and $\delta(\mathrm{f})$ values. Thus, this approach it is not limited in terms of the choice of $\delta(\mathrm{A})$ and $\delta(\mathrm{f})$. Finally, with these details, it is straight forward to define the values of SH 
in terms of $\mathrm{r}_{\mathrm{FM}}$ and $\mathrm{r}_{\mathrm{AM}}$. In particular, Fig. 2 shows that $\mathrm{FM}$ has a larger $\mathrm{SH}$ than $\mathrm{AM}$ both in the non-contact mode and in the repulsive regime. The numerical results are: $\mathrm{r}_{\mathrm{FM}} \approx 10$ and $23 \mathrm{~nm}$ (outlined markers) and $\mathrm{r}_{\mathrm{AM}} \approx 2$ and $5 \mathrm{~nm}$ (filled markers) for $\mathrm{R}=5$ (squares) and 20 (triangles) $\mathrm{nm}$ respectively in the non-contact mode (Fig. 2a). In the repulsive regime (Fig. $2 \mathrm{~b}$ ) the values are: $\mathrm{r}_{\mathrm{FM}} \approx 6$ and $14 \mathrm{~nm}$ and $\mathrm{r}_{\mathrm{AM}} \approx 4$ and $8 \mathrm{~nm}$ for $\mathrm{R}=5$ and $20 \mathrm{~nm}$ respectively; the markers are used similarly. This example demonstrates that FM is more sensitive to interactions with atoms that are farther away from the tip than AM. In summary, the interactions caused by atom-atom pairs that are at a radial distance $r_{A M}<r<r_{F M}$ affect the frequency shift in the detectable region, i.e. $\left|f(r)-f_{r e f}\right|>\delta(f)$, while the amplitude shifts lie in the non-detectable region here, i.e. $\left|A(r)-A_{\text {ref }}\right|<\delta(A)$ for $r_{A M}<r<r_{F M}$. That is, since $r_{F M}>r_{A M}, A M$ is sensitive only to the interactions with atoms that are directly under the tip, i.e. $r<r_{A M}$, when compared to FM, i.e. $r<r_{F M}$. 

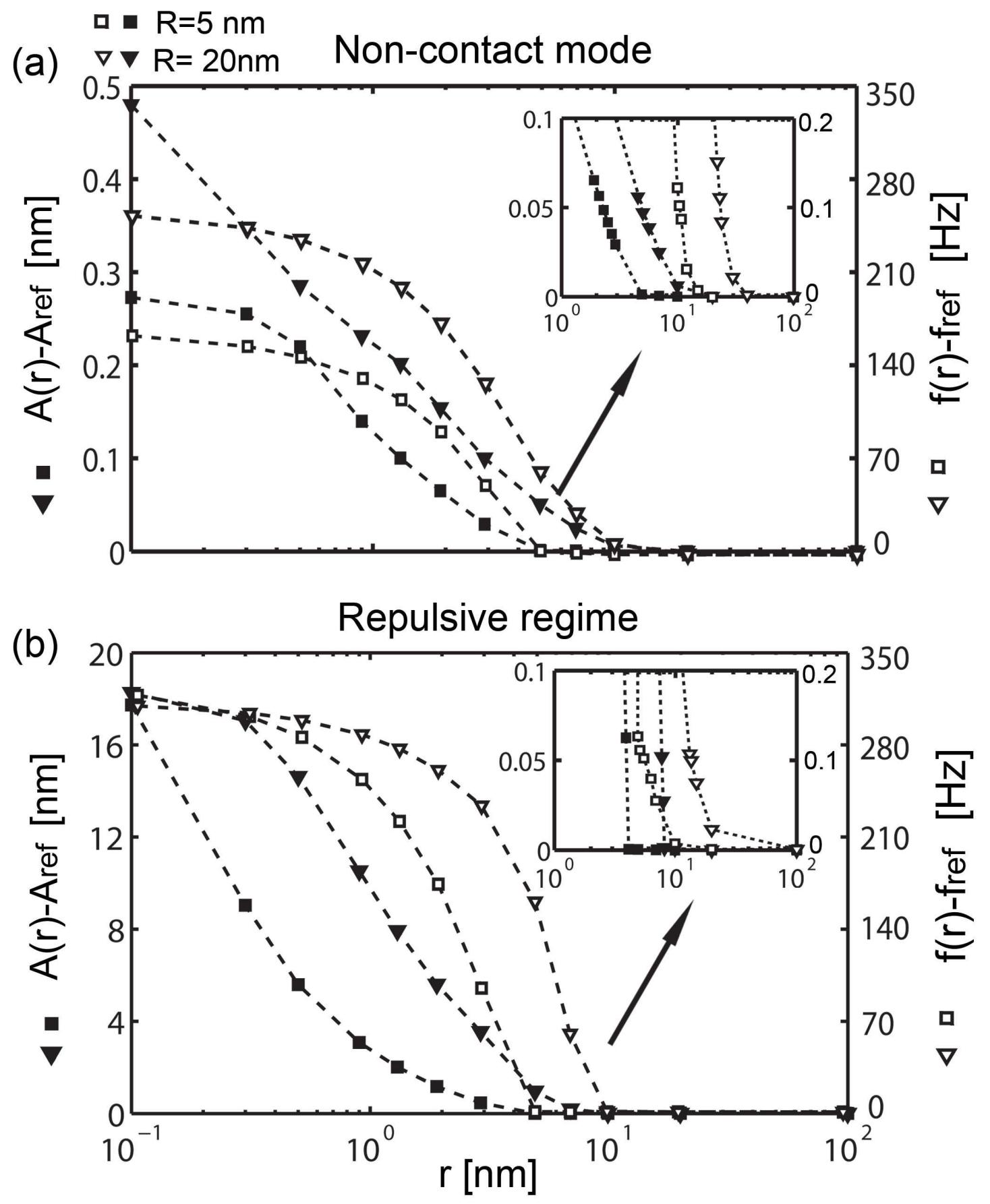

Figure 2. Shifts in amplitude (filled markers) and frequency (outlined markers) due to interactions with atoms lying at radial distance $r$ or less in the (a) non-contact mode and (b) the repulsive regime. The sensitive regions to amplitude and frequency are more easily seen in the insets in (a) and (b) respectively. It is observed that FM is more sensitive to tip-sample interactions that occur at farther radial distances $r$. That is, $\mathrm{r}_{\mathrm{FM}}>$ $\mathrm{r}_{\mathrm{AM}}$. 
The concept of SH can also be used to interpret single atom, or single defect, detection. An example is given next using the non-contact mode of operation. Recall that, so far, the SH has been defined as the limiting space boundary beyond which collective atomic interactions do not affect the detection parameters of a given feedback system. However, in the present case, a single atom, with an interatomic distance $0.2 \mathrm{~nm}$, will be probed. For this purpose this atom is given twice the lattice interatomic strength. The goal is to produce a profile for the contrast, or shifts in amplitude and frequency, due to the presence of the single atom as it approaches the tip from infinity in the radial direction $\mathrm{r}$ (Fig. 3a-b). The interatomic strength can be increased via the parameter $\mathrm{C}$. From the definition of Hamaker $\mathrm{H}=C \pi^{2} \rho_{1} \rho_{2}$ where $\rho_{1}$ and $\rho_{2}$ are the volumetric atomic densities of the two interacting bodies and $\mathrm{C}$ accounts for the strength of the London dispersion interatomic interaction ${ }^{14,19}$. This atom is then added to the lattice and termed the distinct atom on the lattice, i.e. that atom for which $\mathrm{C}$ has been doubled (see Fig. 3b). The addition of this atom results in single atom Spacial Horizons ( $\mathrm{saSH}$ ) with radii $\left(r_{\mathrm{AM}}\right)_{\mathrm{sa}}$ and $\left(\mathrm{r}_{\mathrm{FM}}\right)_{\mathrm{sa}}$ respectively. In Fig. 3 the same parameters as those in Fig. 2a have been used to calculate $A(r)-A_{\text {ref }}$ and $f(r)-f_{\text {ref }}$ respectively. Again, $R=5$ (squares) and $R=$ 20 (triangles) $\mathrm{nm}$ and filled and outlined markers correspond to AM and FM. However, now, $A(r)$ and $f(r)$ make reference to the position of the distinct atom relative to the atom directly under the tip. That is, no atoms are removed from the surface to calculate $\mathrm{A}(\mathrm{r})$ and $\mathrm{f}(\mathrm{r})$. Instead, the position $\mathrm{r}$ of the distinct atom is varied and the variations in amplitude and frequency $\mathrm{A}(\mathrm{r})$ and $\mathrm{f}(\mathrm{r})$ relative to the surface when the distinct atom is not present, i.e. $A_{\text {ref }}$ and $f_{\text {ref, }}$ are recorded as $A(r)-A_{\text {ref }}$ and $f(r)-f_{r e f}$. Thus, the latter are the sources of contrast due to the presence of the distinct atom. Several conclusions follow from this figure. First, note that, in $\mathrm{AM}, \mathrm{A}(\mathrm{r})-\mathrm{A}_{\text {ref }}$ lies in the order of pm even when the distinct atom is right under the tip, i.e. $r=0$. This implies that $A M$ cannot 
resolve the atom due to limitations in the minimum value of detectable amplitude variations $\delta(\mathrm{A})$. That is, $\left(\mathrm{r}_{\mathrm{AM}}\right)_{\mathrm{sa}}$ cannot be defined since $\mathrm{A}(0)-\mathrm{A}_{\mathrm{ref}}<<\delta(\mathrm{A})=0.05 \mathrm{~nm}$ (Fig. 3d). For frequency shift detection, i.e. FM, this is not a problem. That is, in FM, sufficient contrast is guaranteed since $f(0)-f_{\text {ref }}>>(f)=0.1 \mathrm{~Hz}$ (Fig. 3c). Note however that because of excessive spatial sensitivity in frequency $\left(\mathrm{r}_{\mathrm{FM}}\right)_{\mathrm{sa}} \approx 1.5$ for $\mathrm{R}=5 \mathrm{~nm}$ and 20 $\mathrm{nm}$. That is, while the true diameter of the distinct atom is $0.2 \mathrm{~nm}$, the result is that $\left(\mathrm{r}_{\mathrm{FM}}\right)_{\mathrm{sa}}>>0.2 \mathrm{~nm}$ (Fig. 3c) implying that the true dimension is not recovered. The physical interpretation is that FM detects the distinct atom even when it is not exactly under the tip. This causes a form of aberration, i.e. aberrant magnification as illustrated in Figs. 3a-b. It is important however to understand whether the relative contrast is different in the AM and FM modes. Relative contrast is independent of technological detection limits like $\delta(\mathrm{A})$ and $\delta(\mathrm{f})$. Thus, for this purpose we need to dispose of the parameters $\delta(\mathrm{A})$ and $\delta(\mathrm{f})$. Then we can provide further insight regarding the contrast mechanisms for resolving single atoms, or single atomic defects (Fig. 3a). Relative contrast analysis can be carried out by normalizing the contrast parameters, i.e. $\left(A(r)-A_{r e f}\right) /\left(A(0)-A_{r e f}\right)$ and $\left(f(r)-f_{r e f}\right) /\left(f(0)-f_{r e f}\right)$. For relative contrast we can define a minimum detectable shift with the above ratios rather than with $\delta(A)$ and $\delta(f)$. Let us set, for example, the limit $\left(A\left(r_{0.2(A M)}\right)-A_{\text {ref }}\right) /\left(A(0)-A_{\text {ref }}\right)=0.2$. Here 0.2 simply implies that $80 \%$ of the contrast is attenuated at $\mathrm{r}_{0.2(\mathrm{AM})}$ relative to $\mathrm{r}=0$ (see for example the limits in Fig. 3a at 0.2 for $r_{0.2(\mathrm{FM})}$ ). Thus, this defines a radius $\mathrm{r}_{0.2(\mathrm{AM})}$ as the mechanism for resolving single atoms in AM with sufficient topographic contrast. Then, from the inset in Fig. $3 \mathrm{~d}$, if the tip is sharp, i.e. $\mathrm{R}=5 \mathrm{~nm}$ (filled squares), and if $\delta(\mathrm{A})$ could be lowered down to the pm region, the distinct atom could be potentially mapped with nanometer apparent width, i.e. $\mathrm{r}_{0.2(\mathrm{AM})} \approx 1 \mathrm{~nm}$. This value is shown to be similar in AM (filled markers in the inset in Fig. 3d) and FM, i.e. $\mathrm{r}_{0.2(\mathrm{FM})} \approx 1 \mathrm{~nm}$ (outlined markers in Fig. $3 \mathrm{c}$ ); 
$\mathrm{r}_{0.2(\mathrm{FM})}$ is defined in a similar way to $\mathrm{r}_{0.2(\mathrm{AM})}$. In terms of relative contrast, changes in tip radius also affect $\mathrm{AM}$ and $\mathrm{FM}$ similarly (compare squares to triangles in the insets in Figs. 3c-d). Note that the size of the tip is very important in terms of relative contrast (Figs. 3c-d). That is, aberrant magnification dramatically increases with R. Aberrant magnification should further compromise contrast when imaging a true heterogeneous sample where single atoms, or nanostructures, differ in chemistry but are close enough to each other as to lie in the saSH (blue colored regions in Fig. 3b). 

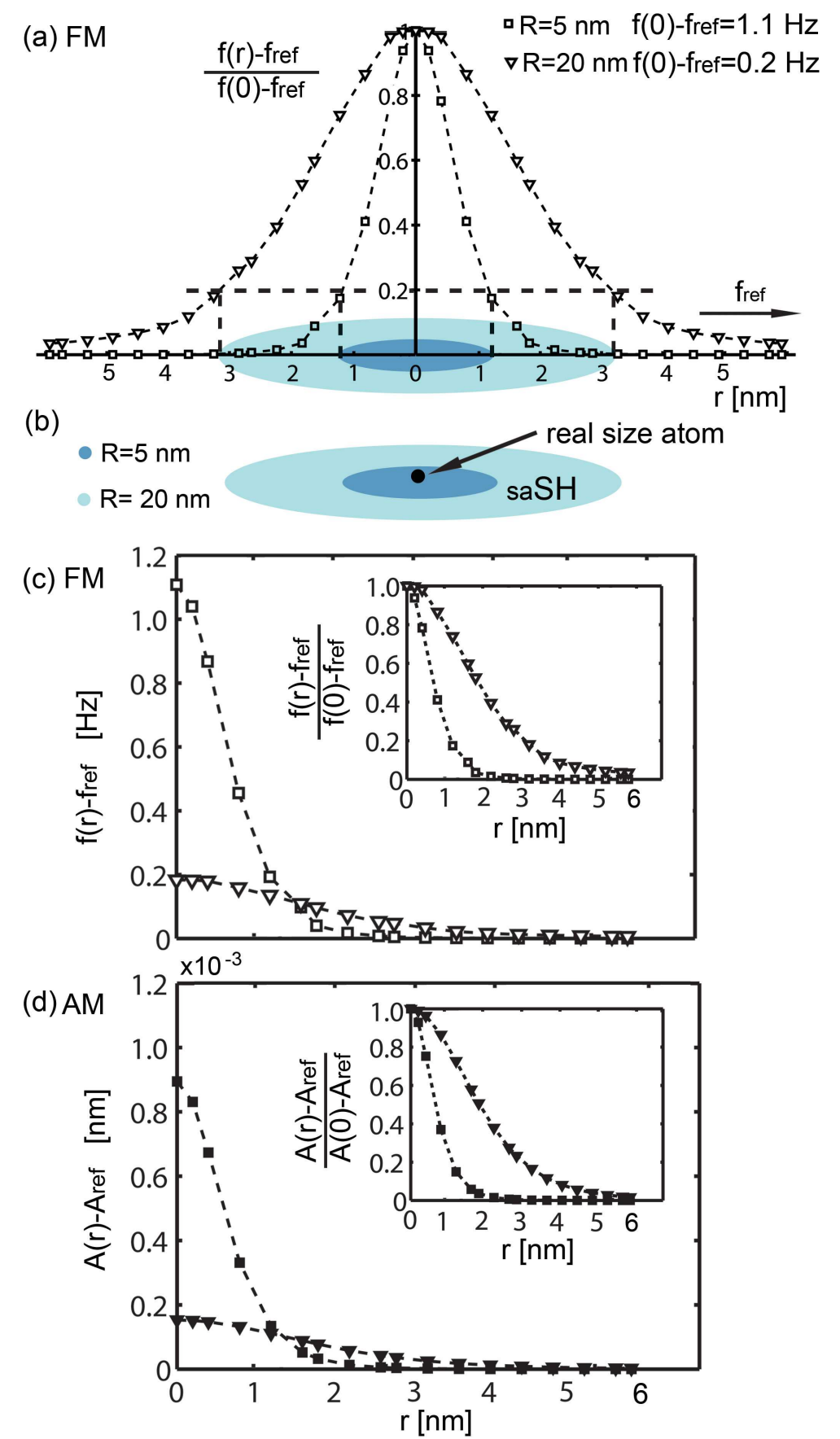

Figure 3. (a) Scheme of the relative contrast produced by an FM system when detecting a single atom of diameter $0.3 \mathrm{~nm}$. (b) The fact that the dynamics are sensitive to interactions form atoms that are at a distance $\mathrm{r}$ larger than $0.3 \mathrm{~nm}$ lead to a single atom $\mathrm{SH}$ (saSH) (light colored) larger than that of the true interatomic distance of the atom. This leads to aberrant magnification. The saSH increases with tip radius R. Absolute and relative (inset) sensitivity when imaging one atom in (c) FM and (d) AM. In absolute terms an AM system might not detect the presence of an atom while in relative terms (insets) the contrast might be the same as that generated by an FM system. 


\section{Conclusions}

The concept of spatial horizon SH has been introduced and used to quantify differences in sensitivity for AM and FM feedback systems. The $\mathrm{SH}$ is the effective area of the sample where detectable tip-sample interactions occur. Ambient conditions have been used for simplicity and because, in ambient, it is relatively easy to operate both instruments from a technological point of view ${ }^{6}$. The results have shown that FM has a larger value of SH implying that detectable frequency shifts partially originate from interactions with atoms that lie relatively far from the tip. This effect is directly attributable to the great sensitivity of FM systems to detect frequency shifts of fractions of a Hertz. This can be relatively easily achieved with an FM controller since both electronic and thermal limits lie below one Hertz ${ }^{6}$. An AM feedback, on the other hand, has greater limitations in terms of amplitude detection because thermal noise lies far above the picometer range (in amplitude) and amplitude detection methods do not have such precision. For example, in terms of single atoms, or single atomic defect detection, we have shown that contrast in frequency shifts are, in principle, readily detectable in an FM system even with the use of relatively large tip curvatures. For the same conditions, in AM, one would have to detect amplitude shifts down to the pico meter range. Nevertheless, our study indicates that relative contrast is equivalent for $\mathrm{AM}$ and FM in terms of single atom detection. That is, if the amplitude shift detection was not limited, then the contrast produced by a single atom, and the corresponding resolution, would be identical in terms of frequency and amplitude shifts. The fact that the $\mathrm{SH}$, or saSH (for single atom detection), is finite, leads to aberrant magnification where the size of a single atom might be reconstructed and displayed, by both AM and FM systems, with dimensions which are much larger than true. In the interpretation 
given in this work, this increase in dimension originates from the interaction between the tip and atoms that are relatively far from the atom directly under the tip.

\section{Methods}

Modeling AM AFM. The AM AFM feedback system has been modeled with the use of Matlab and Simulink as described elsewhere ${ }^{20}$.

Modeling FM AFM. The FM AFM feedback system has been modeled with the use of Matlab and Simulink. Our block diagram is similar to those used in standard modern FM instrumentation as described in the literature ${ }^{2,6}$.

Modeling discrete tip-sample forces from continuous models. In our model we have only used conservative forces, i.e. long range van der Waals (vdW) and short range repulsive as described in the main text. Nevertheless, since these forces are fundamental and ever prevailing in any nanoscale interaction ${ }^{6}$, our study can be seen as a foundation to future AM/FM comparisons. We have discretized the long range vdW forces by taking an interatomic distance of $0.2 \mathrm{~nm}$ per atom and forcing the value of the net force, as it reaches infinity in the radial direction, i.e. infinite atoms, to match the standard continuum vdW derivation ${ }^{19}$. In the standard derivation the Hamaker constant is used and an infinite number of atoms are accounted for. 
1. Binnig, G.; Quate, C. F.; Gerber, C., Atomic Force Microscope. Physical Review Letters 1986, 56, 930-933.

2. Giessibl, F. J., Advances in atomic force microscopy Reviews of Modern Physics 2003, 75, 949-983.

3. Gan, Y., Atomic and subnanometer resolution in ambient conditions by atomic force microscopy. Surface Science Reports 2009, 64, 99-121.

4. Ohnesorge, F.; Binnig, G., True Atomic Resolution by Atomic Force Microscopy Through Repulsive and Attractive Forces. Science 1993, 260, 1451-1456

5. Fukuma, T.; Kobayashi, K.; Matsushige, K.; Yamada, H., True molecular resolution in liquid by frequency modulation atomic force microscopy. Applied Physics Letters 2005, 86, 193108-193110.

6. Garcia, R.; Perez, R., Dynamic Atomic Force Microscopy Methods. Surface Science Reports 2002, 47 197-301.

7. San Paulo, A.; Garcia, R., High-Resolution Imaging of Antibodies by TappingMode Atomic Force Microscopy: Attractive and Repulsive Tip-Sample Interaction Regimes. Biophysical Journal 2000, 78, 1599-1605.

8. Albrecht, T. R.; Grutter, P.; Horne, D.; Rugar, D., Frequency modulation detection using high - Q cantilevers for enhanced force microscope sensitivity. Journal of Applied Physics 1991, 69, 668-673.

9. Yang, C.-W.; Hwang, I.-S.; Fu Chen, Y.; Seng Chang, C.; Ping Tsai, D., Imaging of soft matter with tapping-mode atomic force microscopy and non-contactmode atomic force microscopy. Nanotechnology 2007, 18, 084009.

10. Garcia, R., Amplitude Modulation Atomic Force Microscopy. Wiley-VCH: Weinheim, 2010.

11. Klinov, D.; Magonov, S., True molecular resolution in tapping-mode atomic force microscopy with high-resolution probes. Applied Physics Letters 2004, 84, 2697 2699.

12. Voïtchovsky, K.; Kuna, J. J.; Antoranz Contera, S.; Tosatti, E.; Stellacci, F., Direct mapping of the solid-liquid adhesion energy with subnanometre resolution. Nature Nanotechnology 2010, 5, 401 - 405.

13. Gross, L.; Mohn, F.; Moll, N.; Liljeroth, P.; Meyer, G., The Chemical Structure of a Molecule Resolved by Atomic Force Microscopy. Science 2009, 325, 1110-1114.

14. Israelachvili, J., Intermolecular \& Surface Forces. 2 ed.; Academic Press: 1991.

15. Rodríguez, T. R.; García, R., Tip motion in amplitude modulation tapping-mode atomic-force microscopy: Comparison between continuous and point-mass models. Applied Physics Letters 2002, 80, 1646-1648.

16. Gauthier, M.; Pérez, R.; Arai, T.; Tomitori, M.; Tsukada, M., Interplay between Nonlinearity, Scan Speed, Damping, and Electronics in Frequency Modulation AtomicForce Microscopy. Physical Review Letters 2002, 89, 146104.

17. Garcia, R.; San Paulo, A., Attractive and repulsive tip-sample interaction regimes in tapping mode atomic force microscopy Physical Review B 1999, 60, 49614967.

18. Derjaguin, B. V.; Muller, V.; Toporov, Y., Effect of Contact Deformations on the Adhesion of Particles. Journal of Colloid and Interface Science 1975, 53, 314-326.

19. Hamaker, H. C., The London - van der Waals attraction between spherical particles. Physica 1937, 4, 1058-1072.

20. Santos, S.; Barcons, V.; Verdaguer, A.; Font, J.; Thomson, N. H.; Chiesa, M., How localised are energy dissipation processes in the nanoscale? . Nanotechnology 2011, 22, 345401-345407. 